\title{
Gauge Unification and the Supersymmetric Light Higgs Mass
}

\author{
Jose Ramón Espinosa ${ }^{1}$ and Mariano Quirós ${ }^{2}$ \\ ${ }^{1}$ CERN TH-Division, CH-1211 Geneva 23, Switzerland \\ ${ }^{2}$ IFAE, UAB 08193 Bellaterra, Barcelona, Spain \\ and IEM, CSIC Serrano 123, 28006 Madrid, Spain
}

(Received 6 April 1998)

\begin{abstract}
We consider general supersymmetric models with (a) arbitrary matter content and (b) gauge coupling unification near the string scale $\sim 10^{17} \mathrm{GeV}$, and derive the absolute upper limit on the mass of the lightest Higgs boson. For models without Higgs couplings to extra SU(2) triplets the bound is about $155 \mathrm{GeV}$. The bound is maximized for models with such triplets and can be as high as $205 \mathrm{GeV}$, significantly larger than previous estimates partly due to a normalization error. [S0031-9007(98)06664-2]
\end{abstract}

PACS numbers: 12.60.Jv, 12.10.Kt, 14.80.Cp

Low-energy supersymmetry [1] is a key ingredient in the best-qualified candidate models to supersede the standard model (SM) at energies beyond the $\mathrm{TeV}$ range. The extensive experimental search of the (super-) partners of SM elementary particles predicted by supersymmetry (SUSY) has been unsuccessful so far, challenging [2], with the rise of experimental mass limits, the naturalness and relevance of SUSY for the electroweak-scale physics.

In this context, the sector of the theory responsible for electroweak symmetry breaking has a special status. While all superpartners of the known SM particles can be made heavy by simply rising soft SUSY-breaking mass parameters in the model, the Higgs sector necessarily contains a physical Higgs scalar whose mass does not depend sensitively on the details of soft masses but is fixed by the scale of electroweak symmetry breaking. This important fact follows simply from the spontaneous breaking of the electroweak gauge symmetry $[3,4]$ and it is not specific to supersymmetric models. More precisely, the general statement is that some Higgs boson must exist whose mass squared satisfies $m_{h}^{2} \leq \lambda v^{2}$, where $v$ is the electroweak scale $(v=174.1 \mathrm{GeV})$ and $\lambda$ is the dimensionless quartic coupling of some Higgs state in the model. In other words, the mass of the light Higgs can be made heavy only at the expense of making the coupling $\lambda$ very strong. The role of supersymmetry is to fix $\lambda$ in some models. For example, in the minimal supersymmetric standard model (MSSM), which includes two Higgs doublets,

$$
H_{1}=\left(\begin{array}{c}
H_{1}^{o} \\
H_{1}^{-}
\end{array}\right) \text {and } H_{2}=\left(\begin{array}{c}
H_{2}^{+} \\
H_{2}^{o}
\end{array}\right),
$$

to give masses to quarks and leptons, $\lambda$ is related to the $\mathrm{SU}(2)_{L} \times \mathrm{U}(1)_{Y}$ gauge couplings ( $g$ and $g^{\prime}$, respectively) and the following (tree-level) bound on the mass of the lightest Higgs boson holds

$$
m_{h}^{2} \leq M_{Z}^{2} \cos ^{2} 2 \beta,
$$

where $\tan \beta=\left\langle H_{2}^{0}\right\rangle /\left\langle H_{1}^{0}\right\rangle$. This represents a very stringent prediction which, as is well known, gets significantly relaxed when radiative corrections to $\lambda$ are included [5-
7]. These corrections depend logarithmically on the soft masses and push the upper mass limit for the lightest Higgs boson of the MSSM up to $125 \mathrm{GeV}$ (for a top-quark mass $M_{t}=175 \mathrm{GeV}$ and $M_{\mathrm{SUSY}}<1 \mathrm{TeV}$ ).

The fact that $\lambda$ is calculable in terms of gauge couplings in the MSSM is due to supersymmetry and to the fact that the superpotential does not contain cubic terms of the form $W=h_{X} X H_{i} H_{j}$ (with $i, j=1,2$ ) as no $X$ field exists with the appropriate quantum numbers to form a gauge-invariant object. In extended models, the presence of such fields and couplings modifies the quartic Higgs self-interactions, which can ultimately have an impact on the tree-level upper bound on $m_{h}^{2}$, which receives corrections proportional to $h_{X}^{2} v^{2}$. The Yukawa coupling $h_{X}$ is unknown but asymptotically nonfree, and so it can be bounded from above if it is further required to remain in the perturbative regime up to some large energy scale [where grand unified theory (GUT), string, or Planck physics takes over].

As there is no reason to believe that low-energy supersymmetry is realized in Nature in the form of the MSSM, the experimentalist willing to test SUSY via Higgs searches would like to know what is the absolute general upper limit that should be reached on $m_{h}$, so as to rule out low-energy SUSY. This is the important question we set ourselves to answer in this Letter. The new ingredients with respect to our earlier analyses [8,9], yielding upper bounds $\sim 140 \mathrm{GeV}$, are that we maximize the effect of large gauge couplings in the Higgs mass bound, we take into account the difference between running and pole top masses, and use a more precise evaluation of radiative corrections. We also correct a normalization error in our previous results $[8,9]$.

To give a precise answer we have to assume that all couplings in the theory remain perturbative up to a very large energy scale. This is particularly well motivated in low-energy SUSY models from the successful unification of gauge couplings in the simplest MSSM model, the best (indirect) evidence we have so far for SUSY. In considering extensions of the MSSM, we will always require that 
this remarkable feature is not spoiled. The addition of extra gauge singlets or complete $\mathrm{SU}(5)$ representations is the natural possibility for extended models where minimal unification is automatically preserved (at the one-loop level). In our search for the upper Higgs mass limit, we do not restrict ourselves to these possibilities but consider other options. Although split SU(5) representations are difficult to arrange in GUT models, they can easily arise in string models and may even help in solving the mismatch [10] between the MSSM unification scale, $M_{G} \sim 2 \times 10^{16} \mathrm{GeV}$, and the string scale, $M_{S} \sim 5 \times 10^{17} \mathrm{GeV}$ [degenerate full $\mathrm{SU}(5)$ multiplets do not modify (at one loop) the unification scale].

We assume that any model must contain at least the two minimal Higgs doublets $H_{1,2}$ required to give quarks and leptons their masses. In principle, more than two Higgs doublets could be involved in $\mathrm{SU}(2)_{L} \times \mathrm{U}(1)_{Y}$ breaking, but in such a case a rotation in field space can be made so that only $H_{1,2}$ have nonzero vacuum expectation values. Additional higher Higgs representations are generally very constrained by $\rho=M_{W}^{2} / M_{Z}^{2} \cos ^{2} \theta_{W} \simeq 1$. In addition, if they contribute significantly to the $W^{ \pm}$and $Z^{0}$ masses, the Higgs bound, sensitive to the doublet contribution, gets weaker and, in addition, other light Higgses appear in the spectrum [4]. It is thus conservative to assume that $\left\langle H_{1,2}^{0}\right\rangle$ are responsible for all the breaking in which case the known $Z^{0}$ mass fixes $\left\langle H_{1}^{0}\right\rangle^{2}+\left\langle H_{2}^{0}\right\rangle^{2}=v^{2}$.

To maximize the upper bound on $m_{h}$ we next assume that the model also contains extra chiral multiplets with the appropriate quantum numbers to give couplings of the form $W=h_{X} X H_{i} H_{j}$. Thus, $X$ can be only a singlet $(S)$ or a $Y=0, \pm 1$ triplet $\left(T_{Y}\right)$. From the gauge-invariant trilinear superpotential

$$
\begin{aligned}
W= & \lambda_{1} H_{1} \cdot H_{2} S+\lambda_{2} H_{1} \cdot T_{0} H_{2} \\
& +\chi_{1} H_{1} \cdot T_{1} H_{1}+\chi_{2} H_{2} \cdot T_{-1} H_{2},
\end{aligned}
$$

the tree-level mass bound follows (we correct here a normalization error in the $\chi_{1,2}$ terms of Refs. [8,9]):

$$
\begin{aligned}
m_{h}^{2} / v^{2} \leq & \frac{1}{2}\left(g^{2}+g^{2}\right) \cos ^{2} 2 \beta+\left(\lambda_{1}^{2}+\frac{1}{2} \lambda_{2}^{2}\right) \sin ^{2} 2 \beta \\
& +4 \chi_{1}^{2} \cos ^{4} \beta+4 \chi_{2}^{2} \sin ^{4} \beta .
\end{aligned}
$$

The different dependence of the various terms on $\tan \beta$ will make them important in different regimes. In particular we already anticipate that, in the large-tan $\beta$ region, the $\chi_{2}$ contribution will be crucial for the upper limit. $S$ and $T_{0}$ have the same dependence, while it can be shown that the effect of $\lambda_{1}$ is always more important than that of $\lambda_{2}$. For this reason we will not take into account the possible effect of $T_{0}$ representations.

As the next step, one should impose triviality bounds on the extra couplings entering (4) by assuming that they do not reach a Landau pole below the unification scale. As stressed in [11,12], large values of the gauge couplings slow down the running of Yukawa couplings with increasing energy and so they can be larger at the electroweak scale. To get numerical values for these upper bounds we then have to further specify the particle content of the model, imposing gauge-coupling unification and making the gauge couplings as large as possible. For the renormalization group (RG) analysis we consider that all superpartners can be roughly characterized by a common mass $M_{\text {SUSY }}$ below which the effective theory is just the SM and, restricted by naturalness, we take $M_{\text {SUSY }}=$ $1 \mathrm{TeV}$. No such constraint should be imposed on extra matter in vectorlike representations, such as $(5+\overline{5}) \mathrm{SU}(5)$ pairs, which could be present at intermediate scales. By setting their masses down to $1 \mathrm{TeV}$ we are enhancing their effect on the running of gauge couplings, which being stronger will also tend to increase the $m_{h}$ bound.

To achieve unification with only one scale $M_{\text {SUSY }}$ fixed to $1 \mathrm{TeV}$ is not completely trivial. When the MSSM is enlarged by one singlet $S$ and a pair $\left\{T_{1}, T_{-1}\right\}$ (to cancel anomalies) the running $g_{1}^{2}=5 g^{\prime 2} / 3$ and $g^{2}$ meet at $M_{X} \sim 10^{17} \mathrm{GeV}$. Interestingly enough, this is closer to the heterotic string scale than the MSSM unification scale. Of course, $g_{3}^{2}$ fails to unify unless extra matter is added. This can be achieved, for example, by adding four $(3+$ 3) $\left[\mathrm{SU}(2)_{L} \times \mathrm{U}(1)_{Y}\right.$ "singlet quark" chiral multiplets] or $(3+\overline{3})$ plus one $\mathrm{SU}(3)_{c}$ octet. In addition to this, we can still have a $(5+5) \mathrm{SU}(5)$ pair, which will not change the unification scale. The unification of the couplings is shown in Fig. 1 (solid lines).

For comparison, dashed lines show the running couplings when their beta functions are chosen in such a way that all couplings reach a Landau pole at the unification scale. In this case the low-energy couplings are fully determined by the "light" matter content of the model, which determines the RG beta functions. This behavior is dubbed nonperturbative unification [13] and was long ago proposed as an alternative to conventional unification, with

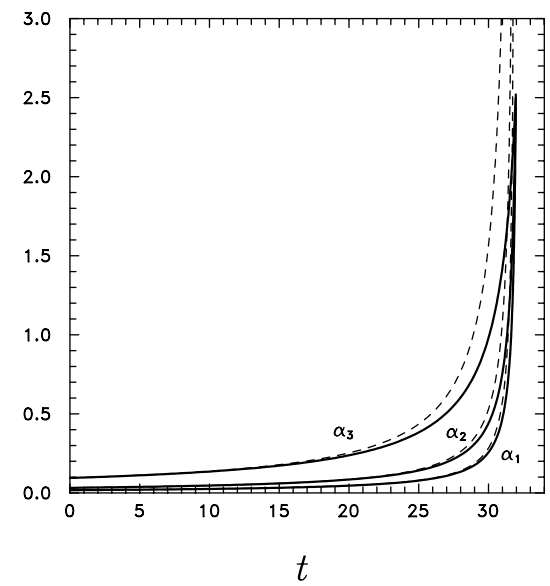

FIG. 1. Running $\alpha_{i}$ 's $\left(=g_{i}^{2} / 4 \pi\right)$ for the model discussed in the text (solid lines) and upper perturbative limit (dashed lines) with $t=\log \left(Q / M_{\text {SUSY }}\right)$. 
the attractive feature of having less sensitivity of the lowenergy couplings to high-energy physics (in addition, the choice of $k_{i}$ normalization factors for gauge coupling unification is now immaterial). The dashed lines can be considered as the perturbative upper limit on the gauge couplings, and comparison with the solid lines shows that our model is close to saturation and represents a concrete realization of the most extreme scenario to maximize the $m_{h}$ bound. The emphasis here should lie in this fact rather than in the plausibility or physics motivation of the model per se.

The particular model we use serves the purpose of illustrating the fact that the Higgs mass bound can be saturated. The model includes exotic representations with noncanonical charge assignment, which can nevertheless appear in string models: $(3,1)_{0}$ and $(\overline{3}, 1)_{0}$ 's can appear in general embeddings [14] of the standard model group other than the usual embeddings in grand unified groups such as $\mathrm{SU}(5)$ or $E_{6}$. On the other hand, $\mathrm{SU}(2)$ triplets are possible if the SU(2) Kač-Moody level is larger than 1 (as we have seen, the effect of this on the unification condition is not important in the limiting case of nonperturbative unification). While these triplets are the key ingredient to go beyond the Higgs mass limits of the MSSM, the additional representations included to ensure unification are not uniquely determined. Different representations of exotic matter at intermediate scales, such as color octets with canonical charge assignment, could equally well give correct unification. In such cases, the lightest Higgs can well be much heavier than in the MSSM, even if the general upper limit is not reached.

Having optimized in this way the most appropriate running gauge couplings, we turn to the running of $\lambda_{1}$ and $\chi_{1,2}$. The relevant RG equations can be found in Refs. $[8,9]$. For a given value of $\tan \beta$ (which influences the top and bottom Yukawa couplings entering the RGs) we find the maximum value of the particular combination of Yukawa couplings that enters the bound (4), compatible with perturbativity up to $M_{S}$. To add the important radiative corrections we follow the RG method, as explained, for instance, in Refs. [15-17], which includes two-loop RG improvement and stop-mixing effects. We fix $M_{t}=175 \mathrm{GeV}$. This physical top-quark mass is related to the Yukawa coupling $h_{t}$ by the modified minimal-subtraction scheme ( $\left.\overline{\mathrm{MS}}\right)$ relation $h_{t} v \sin \beta / \sqrt{2}=M_{t} /\left[1+4 \alpha_{s}(M t) / 3 \pi\right]$.

Before presenting our results, it is worth discussing in more detail the bound presented in Eq. (4). If the extra fields responsible for the enhancement of $m_{h}$ sit at $1 \mathrm{TeV}$, should their effect not decouple from the low-energy effective theory? Indeed, in a simple toy model with an extra singlet $S$ coupled to $H_{1} \cdot H_{2}$ as in (3), when a large supersymmetric mass is given to $S$, the $F$-term contribution $\left(\sim \lambda_{1}^{2}\right)$ to the Higgs doublet self-interactions is canceled by a tree diagram that interchanges the heavy singlet, thus realizing decoupling [18]. If, on the other hand, we lower the mass scale of the extra fields $S$ and $T_{Y}$ to the electroweak scale to avoid decoupling, it is generically the case that more than one light Higgs appear in the spectrum. A complicated mixed squared-mass matrix results whose lightest eigenvalue does not saturate the bound (4). Is then this mass limit simply too conservative an overestimate of the real upper limit? It is easy to convince oneself that, in the presence of soft breaking masses, the perfect decoupling cancellation obtained in the large SUSY mass limit does not take place (we are assuming here that SUSY masses, if present for the extra matter, are not larger than $1 \mathrm{TeV}$ ) and the final lightest Higgs mass depends in a complicated way on these soft mass parameters. The interesting outcome is that soft masses can be adjusted in order to saturate the bound (4); the numbers we will present can thus be reached in particular models and no limits lower than these can be given without additional assumptions (which we will not make here, in the interest of generality).

The final bounds, with radiative corrections included, are presented in Fig. 2. Thick lines are the mass limits when only one Yukawa coupling (either $\lambda_{1}, \chi_{1}$, or $\chi_{2}$ ) is turned on. Solid lines correspond to the case when the particular model described previously is assumed to determine the running of the gauge couplings. Long-dashed lines are instead obtained when all gauge couplings reach a Landau pole at $M_{X} \sim 10^{17} \mathrm{GeV}$ and show that the particular model we used practically saturates the absolute bounds. The thin line is the enveloping of absolute mass limits when all Yukawa couplings are turned on.

Below $\tan \beta \sim 1$ (beyond $\tan \beta \sim 60$ ), $h_{t}\left(h_{b}\right)$ reaches a Landau pole below $M_{X}$ and that region is thus excluded. Lines labeled " $\lambda_{1}$ " show the effect of the singlet coupling $\lambda_{1}$. The maximal value of this coupling depends on $\tan \beta$ through $h_{t}$. For small $\tan \beta, h_{t}$ is large and forces $\lambda_{1}$ to be small. With increasing $\tan \beta, h_{t}$ becomes smaller and $\lambda_{1}$ can get larger values. This effect, combined with the

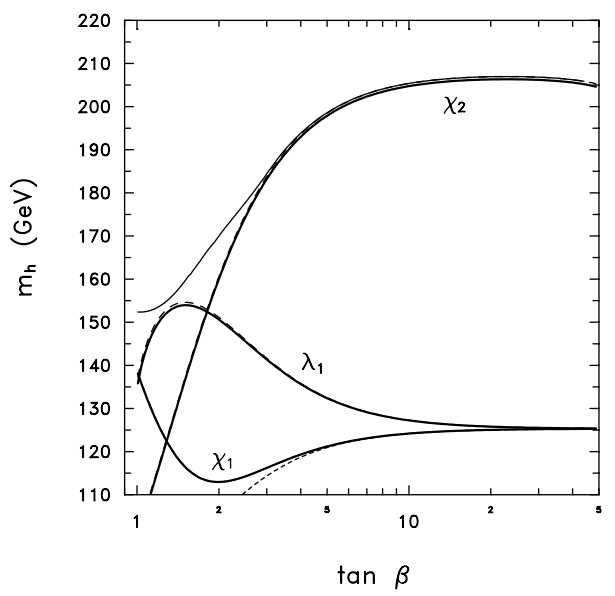

FIG. 2. Radiatively corrected upper bounds on $m_{h}$ when different Yukawa couplings are present in the model and for different assumptions on the running gauge couplings. The short-dashed line gives the upper bound in the MSSM. The thin line is the enveloping of absolute upper bounds. 
$\sin ^{2} 2 \beta$ dependence of the $\lambda_{1}$ contribution to $m_{h}^{2}$ explains the shape of these lines. At large $\tan \beta$, the $\lambda_{1}$ contribution to the mass limit shuts off and the MSSM limit is recovered. [A limit similar to the $\lambda_{1}$ bound has recently been obtained [12] in the MSSM with a singlet field $S$ and pairs of SU(5) $(5+\overline{5})$ saturating the gauge couplings.] The same happens for the lines labeled " $\chi_{1}$," which give the $\chi_{1}$ bound. The upper triviality bound on this Yukawa coupling is basically independent of $\tan \beta$. The interplay between the minimal $\left(\cos ^{2} 2 \beta\right)$ piece of the bound (4) and the $\chi_{1}$ piece explains the shape of these lines. It is interesting how the effect of the $\chi_{2}$ coupling is instead more important for large $\tan \beta$, where it reinforces the minimal contribution, providing the absolute upper limit. This is given by the lines labeled " $\chi_{2}$ " and can be as large as $205 \mathrm{GeV}$.

In conclusion, we calculate a numerical absolute upper limit on the mass of the lightest supersymmetric Higgs boson for any model with arbitrary matter content compatible with gauge coupling unification around (and perturbativity up to) the string scale. For models without Higgs couplings to extra SU(2) triplets the bound is about $155 \mathrm{GeV}$. Previous calculations for models including such triplets $[8,9]$ missed a factor of 4 in the normalization of the additional contribution to the tree-level squared Higgs mass, and we find the bound in such models can be as high as $205 \mathrm{GeV}$, significantly heavier than previously thought $[8,9,11]$. The model saturating this bound has asymptotically divergent gauge couplings and points toward nonperturbative unification. Besides being of obvious interest to the experimentalists, this result has interest for theorists too. If Higgs searches reach the MSSM bounds without finding a signal for a Higgs boson, this could be taken, if one is willing to stick to low-energy supersymmetry, as evidence for additional matter beyond the minimal model. Without stressing the point too much, this could be welcome to reconcile the unification scale with the string scale.

[1] H.-P. Nilles, Phys. Rep. 110, 1 (1984); H. E. Haber and G. L. Kane, Phys. Rep. 117, 75 (1985); R. Barbieri, Riv. Nuovo Cimento 11, 1 (1989).

[2] See, e.g., P. Chankowski, J. Ellis, and S. Pokorski, Phys. Lett. B 423, 327 (1998); R. Barbieri and A. Strumia, hep$\mathrm{ph} / 9801353$.
[3] P. Langacker and H. A. Weldon, Phys. Rev. Lett. 52, 1377 (1984); H. A. Weldon, Phys. Lett. 146B, 59 (1984).

[4] D. Comelli and J.R. Espinosa, Phys. Lett. B 388, 793 (1996).

[5] H. E. Haber and R. Hempfling, Phys. Rev. Lett. 66, 1815 (1991); A. Yamada, Phys. Lett. B 263, 233 (1991); P. H. Chankowski, S. Pokorski, and J. Rosiek, Phys. Lett. B 274, 191 (1992).

[6] Y. Okada, M. Yamaguchi, and T. Yanagida, Prog. Theor. Phys. 85, 1 (1991); J. Ellis, G. Ridolfi, and F. Zwirner, Phys. Lett. B 257, 83 (1991); 262, 477 (1991); R. Barbieri and M. Frigeni, Phys. Lett. B 258, 395 (1991); A. Brignole, J. Ellis, G. Ridolfi, and F. Zwirner, Phys. Lett. B 271, 123 (1991); A. Brignole, Phys. Lett. B 281, 284 (1992).

[7] Y. Okada, M. Yamaguchi, and T. Yanagida, Phys. Lett. B 262, 54 (1991); R. Barbieri, M. Frigeni, and F. Caravaglios, Phys. Lett. B 258, 167 (1991); J. R. Espinosa and M. Quirós, Phys. Lett. B 266, 389 (1991).

[8] J. R. Espinosa and M. Quirós, Phys. Lett. B 279, 92 (1992).

[9] J. R. Espinosa and M. Quirós, Phys. Lett. B 302, 51 (1993).

[10] K. Dienes, Phys. Rep. 287, 447-525 (1997).

[11] G. L. Kane, C. Kolda, and J. D. Wells, Phys. Rev. Lett. 70, 2686 (1993).

[12] M. Masip, R. Muñoz-Tapia, and A. Pomarol, Phys. Rev. D 57, 5340 (1998).

[13] L. Maiani, G. Parisi, and R. Petronzio, Nucl. Phys. B136, 115 (1978); L. Maiani and R. Petronzio, Phys. Lett. B 176, 120 (1986); N. Cabibbo and G. R. Farrar, Phys. Lett. 110B, 107 (1982); M. Lanzagorta and G. G. Ross, Phys. Lett. B 364, 319 (1995); D. Ghilencea, M. Lanzagorta, and G. G. Ross, hep-ph/9707462.

[14] S. Chaudhuri, G. Hockney, and J. Lykken, Nucl. Phys. B469, 357 (1996).

[15] J. Kodaira, Y. Yasui, and K. Sasaki, Phys. Rev. D 50, 7035 (1994); R. Hempfling and A. H. Hoang, Phys. Lett. B 331, 99 (1994); J. A. Casas, J. R. Espinosa, M. Quirós, and A. Riotto, Nucl. Phys. B436, 3 (1995); B439, 466(E) (1995).

[16] M. Carena, J.R. Espinosa, M. Quirós, and C.E.M. Wagner, Phys. Lett. B 355, 209 (1995); M. Carena, M. Quirós, and C.E. M. Wagner, Nucl. Phys. B461, 407 (1996); H.E. Haber, R. Hempfling, and A.H. Hoang, Z. Phys. C 75, 539 (1997).

[17] S. Heinemeyer, W. Hollik, and G. Weiglein, hep-ph/ 9803277.

[18] H. Georgi, in Grand Unified Theories and Related Topics: Proceedings of the 4th Kyoto Summer Institute, Kyoto, Japan, 1981 (World Scientific, Singapore, 1981). 